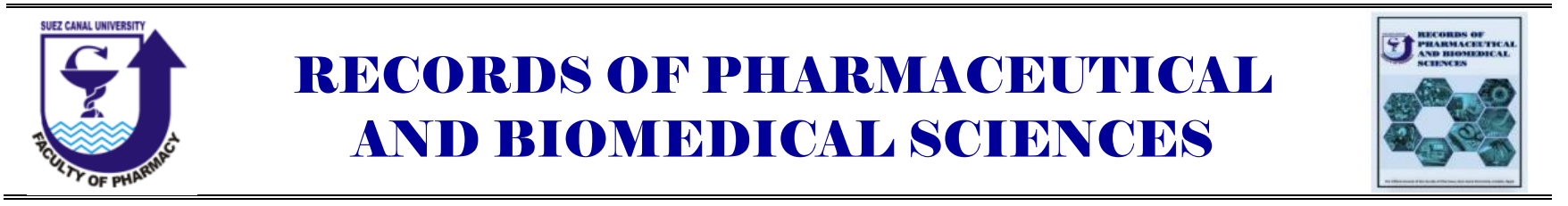

\title{
Implication of LncRNAs MEG3 and LINC00305 in Pathophysiological Mechanisms Associated with Rheumatoid Arthritis
}

\author{
Alaa S. Wahba ${ }^{a^{*}}$, Maha E. Ibrahim ${ }^{b}$, Eman T. Mehanna ${ }^{a}$, Noha M. Mesbah ${ }^{a}$, \\ Samy M. Saleh ${ }^{a}$, Dina M. Abo-elmatty ${ }^{\text {a }}$ \\ ${ }^{a}$ Department of Biochemistry, Faculty of Pharmacy, Suez Canal University, Ismailia, Egypt, ${ }^{b}$ Department of \\ Physical Medicine, Rheumatology and Rehabilitation, Faculty of Medicine, \\ Suez Canal University, Ismailia, Egypt
}

Received on: 14. 06. 2020

Revised on: 16. 07. 2020

Accepted on: 19. 076. 2020

*Correspondence Author:

Tel: +201272823020

E-mail address:

alaa.samir@pharm.suez.edu.eg

\begin{abstract}
Rheumatoid arthritis (RA) is a joint destructive disorder with great morbidity. Early diagnosis and early and effective therapy may prevent joint damage and lead to better long-term results. Therefore, reliable biomarkers and outcome measures are needed. Complex interplay between multiple risk factors contributes to RA. These risk factors include environmental factors, genetic factors and epigenetic modifications. Long non-coding RNAs (lncRNAs) are involved in these epigenetic mechanisms. Accumulating evidence has shown that IncRNAs participate in the processes of inflammation, aberrant proliferation, apoptosis and angiogenesis. They also play roles in autoimmune diseases, such as SLE, Sjogren syndrome, RA and multiple sclerosis. Maternally expressed gene 3 (MEG3) is a maternally expressed lncRNA of the imprinted DLK1-MEG3 locus located on human 14q32 chromosome. It functions as a tumor suppressor. Decreased MEG3 expression has been observed in various human cancers, both type 1 and type 2 diabetes, osteoarthritis and in RA. Decreased serum levels of lncRNA MEG3 in RA could be attributed to MEG3 promoter hypermethylation induced by hypoxia. This downregulation was associated with increased inflammation, cell proliferation and cell invasion and decreased apoptosis. Long intergenic nonProtein coding RNA 00305 (LINC00305) was identified as a pro-inflammatory atherosclerosis-associated IncRNA. RA was associated with increased LINC00305 which served as a regulator of inflammatory, hypoxic, invasive, apoptotic and proliferative mechanisms associated with RA.
\end{abstract}

Keywords: Rheumatoid arthritis; MEG3; LINC00305.

\section{Introduction}

Rheumatoid arthritis (RA) is a systemic inflammatory autoimmune disease triggering joint inflammation combined with extra-articular involvement and profound disability (Guo et al., 2018). The formation of autoantibodies is a typical characteristic of RA specially rheumatoid factors and anti-citrullinated protein antibodies (Alarcon et al., 1982). Advances in understanding the pathogenesis of the disease led to greatly improved RA treatment, emphasizing early intervention soon after diagnosis and escalating the therapy based on disease activity assessment and treatment response in pursuit of clinical remission (Lao and $\mathbf{X u}$, 2020). 
Genetic, environmental factors and epigenetic modifications have been identified to play a role in disease initiation and progression. The human leukocyte antigen (HLA) genes have greater influence on RA. Smoking and infection are environmental factors that affect the development, and severity of RA (Arend and Firestein, 2012). The epigenetics study revealed that DNA methylation, histone modification, microRNAs (miRNAs), long non-coding RNAs (lncRNAs) directly affect genes associated with inflammation and/or tissue destruction. LncRNAs are implicated in a wide range of biological processes from chromatin remodeling to transcriptional and posttranscriptional modifications (Strietholt et al., 2008; Lao and $\mathrm{Xu}, 2020$ ).

\section{Rheumatoid arthritis pathogenesis}

Complex interplay between multiple risk factors is often required leading to initiation and propagation of autoimmunity against modified self-proteins which can occur years before the onset of subclinical synovitis (inflammation of the synovium) and clinical symptoms (Smolen et al., 2016) (Figure 1).

\subsection{Preclinical RA}

The interaction between the previous causative factors triggers protein citrullination and production of autoantibodies. Following citrullination, the altered peptides bind to major histocompatibility complex (MHC) protein heterodimers, especially those containing the shared epitope. This leads to antigen presentation to $\mathrm{T}$ cells, which in turn stimulate $\mathrm{B}$ cells to synthesize a range of antibodies that recognize self-proteins, including $\mathrm{RF}$ (targeting IgGs) and ACPAs (targeting citrullinated proteins). The stage is called loss of tolerance (Muller and Radic, 2015).

\subsection{Early RA}

Clinical synovitis is initiated due to inflammatory cells infiltration into the synovium (Arend and Firestein, 2012). Fibroblast-like synoviocytes (FLSs) interact with the immune cells producing a large set of inflammatory cytokines and chemokines and thereby actively contribute to the inflammatory state in RA owing to activation of the notch and nuclear factor $-\kappa \mathrm{B}(\mathrm{NF}-\kappa \mathrm{B})$ pathways (McInnes and Schett, 2011; Lao and $X u, 2020$ ). $\mathrm{NF}-\mathrm{\kappa B}$ is a major inflammatory mediator functioning in different cell types. NF- $\kappa B$ consists of a group of inducible transcription factors (TFs), which controls multiple genes that play a role in cellular immune and inflammatory processes (Liu et al., 2017).

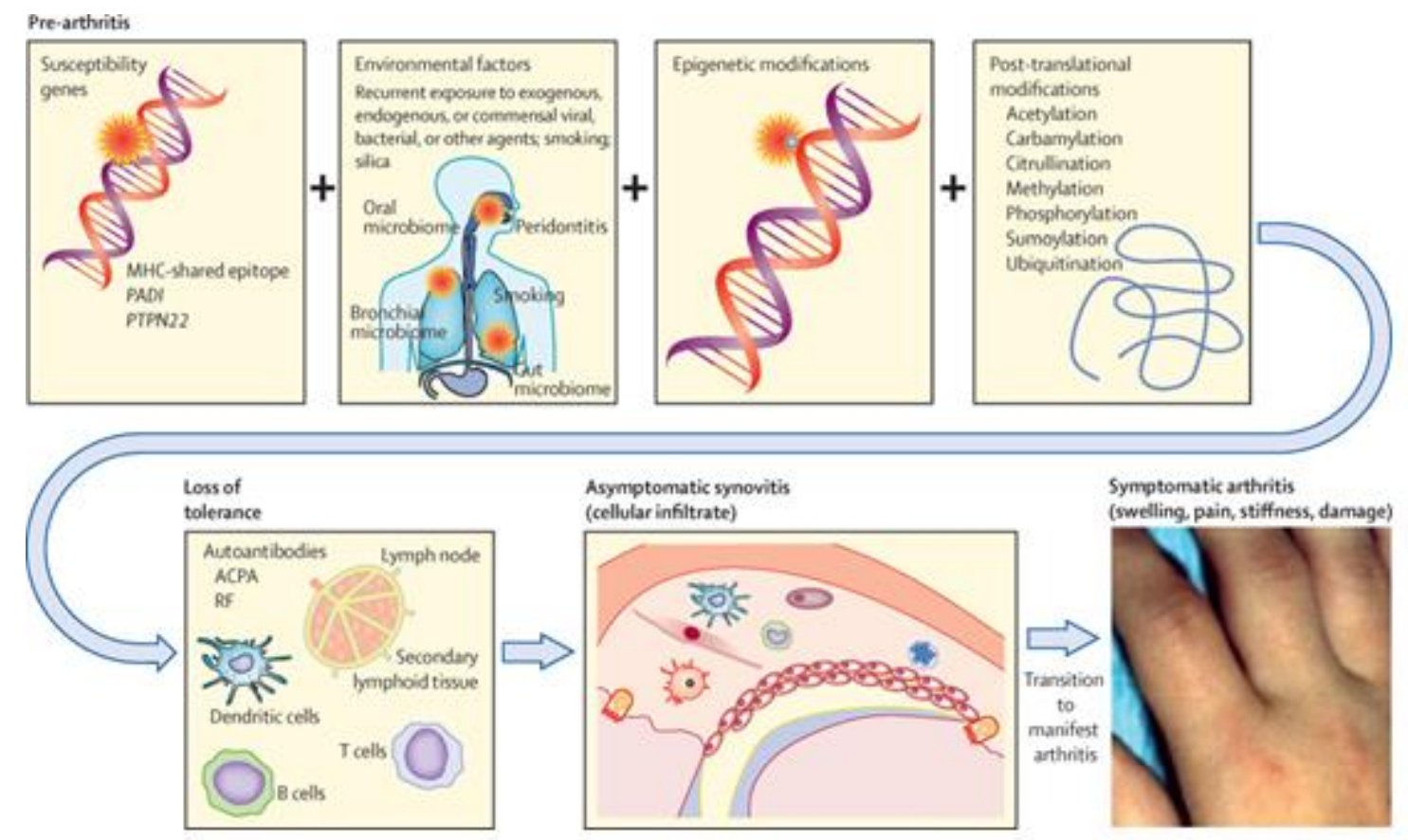

Figure 1: Development and progression of rheumatoid arthritis (Smolen et al., 2016). 


\subsection{Established RA}

Progression to established RA occurs as fibroblastlike synoviocytes (FLSs) secrete cytokines, growth factors and enzymes (matrix metalloproteinases (MMPs) and collagenase), promoting synovial hyperplasia and cartilage destruction (Guo et al., 2018). MMPs are zinc-dependent endopeptidases which mediate basement membrane and extracellular matrix protein degradation, promoting tissue damage. Thus, their enhanced production in RA contributes to loss of cartilage and joint integrity (Ni et al.. 2019). Synovial hyperplasia results from cellular proliferation, influx of cells from the circulation and reduced apoptosis through B-cell lymphoma-2 (Bcl-2) family proteins dysregulation ( $\mathbf{L i}$ and Wan, 2013), leading to inadequate oxygenation and local hypoxia (Chimenti et al., 2015). The main factor mediating the response to hypoxic stress is hypoxia inducible factor (HIF)-1, which transactivates a series of genes such as vascular endothelial growth factor (VEGF) that participates in angiogenesis (Hu et al., 2014).

\section{Long non-coding RNAs function and mechanisms}

The importance of lncRNA molecules in different biological processes is beginning to be appreciated. LncRNAs interact with RNA or DNA through complementary base-pairing and interact with protein via direct interaction (RutenbergSchoenberg et al., 2016). Interactions between lncRNA and RNA, DNA, and proteins enable lncRNAs to regulate gene expression at multiple levels, including transcription, post-transcription, translation, post-translation, and epigenetic modification. LncRNAs were shown to interact with protein coding genes and their transcripts to regulate gene expression. Nuclear IncRNAs interact with chromatin remodelling factors and TFs to regulate the expression of neighbouring or distal genes. Also, they regulate transcription and several other transcriptional events of RNA processing. Cytoplasmic lncRNAs were shown to interfere with post-transcriptional regulation such as mRNA stability and degradation as well as translational regulation of mRNAs (Hadjicharalambous and Lindsay, 2019) (Figure 2).

\section{Long non-coding RNAs and rheumatoid arthritis}

Accumulating evidence has shown that lncRNAs participate in the processes of inflammation, aberrant proliferation, apoptosis and angiogenesis. They also play roles in autoimmune diseases, such as SLE, Sjogren syndrome, RA and multiple sclerosis (Liang et al., 2019). LncRNAs regulate gene transcription via interactions with chromatinmodifying proteins and TFs and they potentially regulate mRNA stability and translation in the cytoplasm (Carrieri et al., 2012; Liu et al., 2015).

\subsection{Long non coding RNA maternally expressed gene 3 (MEG3)}

MEG3 is a maternally expressed lncRNA of the imprinted DLK1-MEG3 locus located on human $14 \mathrm{q} 32$ chromosome. It functions as a tumor suppressor (Benetatos et al., 2011). Imprinted loci comprise both maternally- and paternally expressed genes. Reciprocal parental expression occurs between protein-coding genes and non-coding RNAs through epigenetic modifications such as DNA methylation at imprinting control regions (Barlow, 2011).

The imprinted Delta-like homolog 1(DLK1)MEG3 locus contains paternally expressed proteincoding genes (DLK1, retrotransposon-like protein 1 (RTL1), thyroxine 5-deiodinase (DIO3)), maternally expressed non-coding RNAs consisting of the lncRNA MEG3, several miRNAs and small nucleolar RNAs (da Rocha et al., 2008). Methylation of two differentially methylated regions (DMRs) on the paternal allele, one located upstream of the MEG3 transcription start site (IGDMR), and the other overlapping with the promoter of the MEG3 (MEG3-DMR) maintains reciprocal imprinting (Benetatos et al., 2011).

MEG3 is expressed in many normal tissues. Previous research demonstrated that MEG3 overexpression inhibits proliferation and invasion and induces apoptosis (Zhang et al., 2019). On the other hand, decreased MEG3 expression has been observed in various human cancers (Benetatos et al., 2011), both type 1 and type 2 diabetes (Motterle et al., 2016), osteoarthritis (Su et al., 2015) and in RA (Lu and Qian, 2019). 
Rec. Pharm. Biomed. Sci. 5 (1), 8-17, 2021

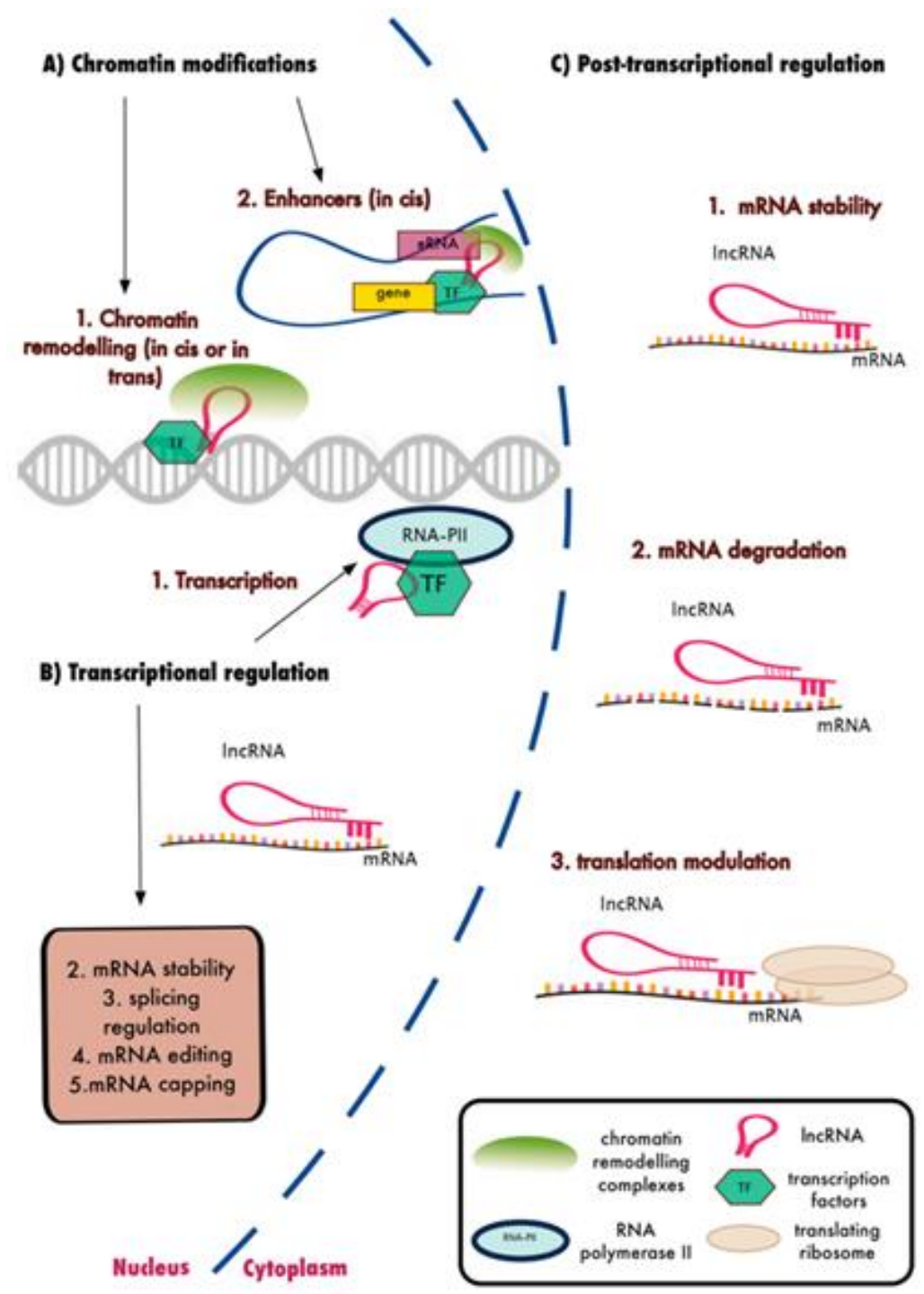

Figure 2: Cellular functions of lncRNAs (Hadjicharalambous and Lindsay, 2019).

Recent studies have demonstrated that the lack of MEG3 expression is involved in RA progression. It was reported that MEG3 promoter hypermethylation might account for its loss in the synovial tissues (Liu et al., 2019). This epigenetic effect on the MEG3 promoter is induced by hypoxia. Hypoxia up-regulates DNA methyltransferases which mediate methyl group transfer to cytosine within a $\mathrm{CpG}$, forming 5methylcytosine. This causes DNA hypermethylation. This hypoxia-induced epigenetic alteration consequently leads to gene silencing by preventing binding of TFs to DNA or by modifying chromatin structure through recruitment of methylDNA binding proteins, thereby making them inaccessible to transcriptional machinery (Hu et al., 2017; Mann, 2017).
Biswas et al. (2019) reported that MEG3 exerts anti-inflammatory activity through NF- $\kappa B$ signaling pathway by specifically targeting important epigenetic regulators, sirtuin (SIRT) 1, miRNA-34a and miRNA-9. MEG3 positively regulates SIRT1 by directly sponging its negative regulator, miRNA-34a and miRNA-9 (Takayama et al., 2009; Cieślik et al., 2020). SIRT 1 inhibits $\mathrm{NF}-\mathrm{kB}$ transcription by deacetylating the RelA/p65 subunit of NF- $\kappa B$ at lysine 310 (Yeung et al., 2004).

MEG3 can repress cell proliferation and angiogenesis (He et al., 2017). Also. It was demonstrated that MEG3 induces apoptosis that is associated with the downregulation of the Bcl-2 protein and the upregulation of the Bax protein (Luo et al., 2015). 
The pro-apoptotic activity together with the antiproliferative activity of lncRNA MEG3 is attributed partly to stimulating the anti-proliferation protein p53 accumulation by blocking p53 degradation through inhibition of murine double minute 2 (MDM2). MEG3 has been shown to interact with p53 and stimulate p53-mediated transcription (AlRugeebah et al., 2019). The p53 protein can negatively regulate VEGFA transcription through binding to its promoter (Qin et al., 2006). Also, MEG3 modulation of Bcl-2/Bax signaling pathways could be attributed to its ability to act as miRNA34a and miRNA-9 sponge, activating SIRT1 which regulates mitochondria-related apoptotic signals (Takayama et al., 2009).

In addition, MEG3 suppresses cell invasion by downregulating the expression of MMP-3, MMP-9 and VEGF. It also reduced epithelial-mesenchymal transition, which is reported to reduce intercellular adhesion and promote cell migration and invasion through negatively regulating miRNA-21 expression which functions as an onco-miRNA (Xu et al., 2018) (Figure 3).

\subsection{Long intergenic non-protein coding RNA 00305 (LINC00305)}

Recent study demonstrated that LINC00305 promotes monocyte inflammation and production of inflammatory cytokines by activating the aryl hydrocarbon receptor repressor (AHRR)-NF- $\mathrm{kB}$ pathway in human monocytes. LINC00305 overexpression has been found in atherosclerotic plaques and monocytes implying its role in monocytes inflammation (Zhang et al., 2017b). Recently, it was demonstrated that RA was associated with increased LINC00305 which served as a regulator of inflammatory, hypoxic, invasive, apoptotic and proliferative mechanisms associated with RA (Wahba et al., 2020).

LINC00305 expression was markedly increased in hypoxia (Zhang et al., 2017a). However, the precise mechanism by which hypoxic conditions affect LINC00305 expression needs further investigation.

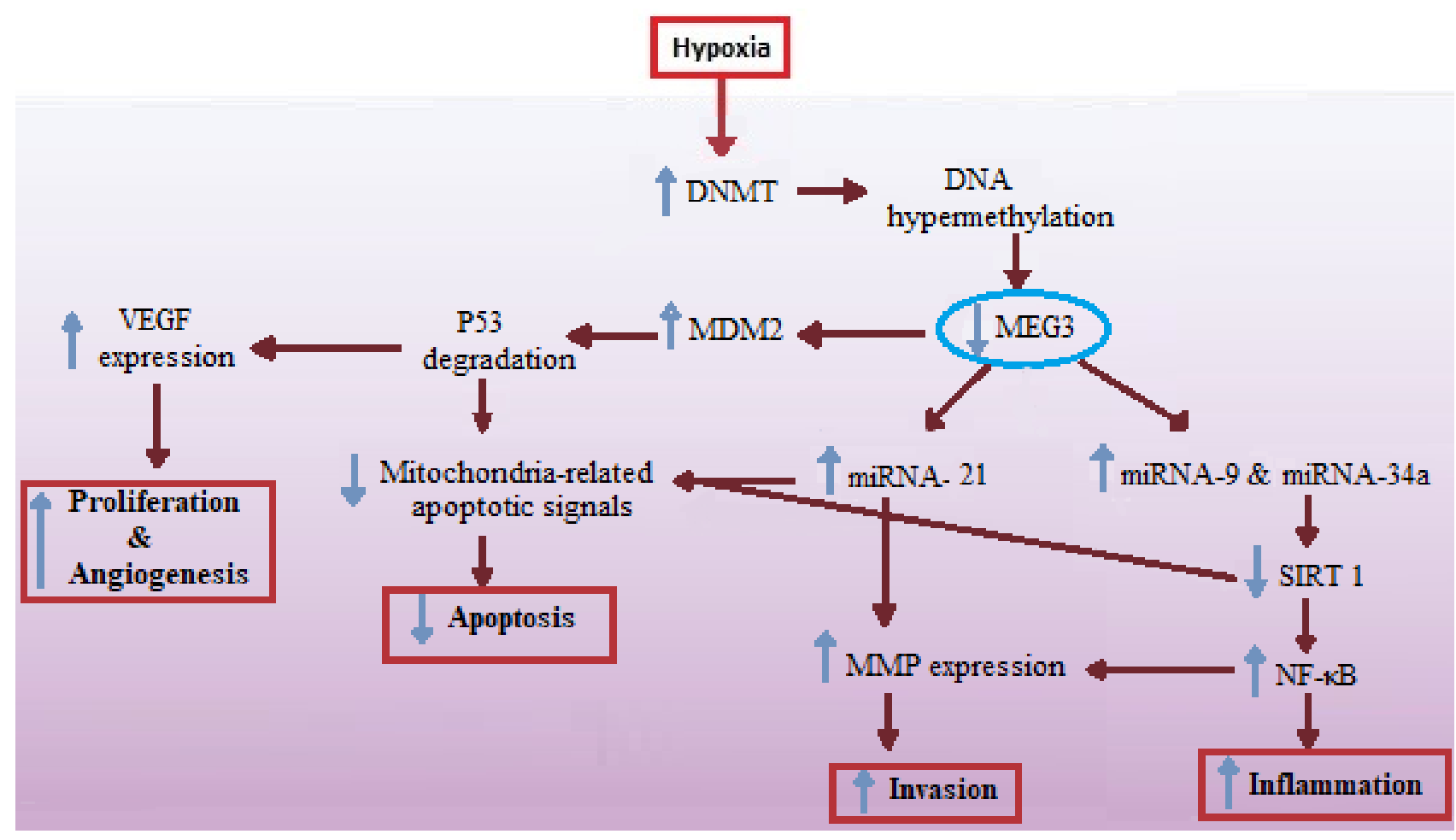

Figure 3: Implication of IncRNA MEG3 in RA pathogenesis. MEG3= maternally expressed gene 3, DNMT= DNA methyltransferases, MDM2 = murine double minute 2, SIRT 1= sirtuin $1, \mathrm{NF}-\kappa \mathrm{B}=$ nuclear factor-kappa beta, MMP $=$ matrix metalloproteinase, VEGF = vascular endothelial growth factor. 
LINC00305 promotes monocyte inflammation and production of inflammatory cytokines by activating the AHRR-NF- $\kappa \mathrm{B}$ pathway in human monocytes. LINC00305 binds to lipocalin-1 interacting membrane receptor (LIMR), promoting the interaction between LIMR and aryl-hydrocarbon receptor repressor (AHRR), the repressor of AHR, leading to enhanced expression and nuclear localization of AHRR which positively regulates NF- $\kappa$ B activity (Zhang et al., 2017b). LINC00305 activates Notch/NF- $\kappa$ B pathway by down-regulating miRNA-124 (Li et al., 2019), whose levels are decreased in RA synoviocytes (Ceribelli et al., 2011). In addition, it was reported that miRNA-124 directly binds to the 3'- UTR of NF- $\kappa B$ p65 inhibiting its expression, thus suppressing proinflammatory cytokine production (Qiu et al., 2015).

MiRNA-124 was confirmed to be involved in the pathogenesis of RA. MiRNA-124 modulates cell growth and invasion by negatively regulating the inhibitory member of the apoptosis stimulating protein p53 (iASPP) which is a key inhibitor of tumor suppressor p53 (Dong et al., 2015). iASPP up-regulation has a confirmed pathogenic role in $\mathrm{RA}$, reduces the expression of $\mathrm{p} 21$, a cell cycle inhibitor regulated by $\mathrm{p} 53$, leading to activation of activator protein-1 (AP-1) which plays a role in the regulation of MMP secretion in RA FLSs (Zeisel et al., 2004; Chen et al., 2017).

Other miRNA-124 target proteins, cyclin-dependent kinase-2 (CDK-2) and monocyte chemoattractant protein-1 (MCP-1), are up-regulated, which leads to increased synovial proliferation, angiogenesis and chemotaxis (Nakamachi et al., 2009). In addition, a previous study hypothesized that miRNA-124 might inhibit the proliferation and invasion of RA synovial fibroblasts (RASFs) through suppressing the expression of NF- $\mathrm{KB}$ ( $\mathbf{L i}$ et al., 2018), which mediates transcriptional activation of several genes including VEGF and MMPs (Okamoto et al., 2007). Another study reported that miRNA-124 inhibited the proliferation and invasion of RASFs through downregulating the expressions of MMP3 (Li et al., 2018). Thus, LINC00305 alters regulation of its target proteins through down-regulating miRNA-124.

It was reported that p53 was down-regulated by iASPP, followed by the up-regulation of antiapoptotic protein $\mathrm{Bcl}-2$ and the down-regulation of pro-apoptotic proteins Bax and caspase-3 (Ma et al., 2017) (Figure 4).

\section{Conclusion}

Accumulating studies have implicated ncRNAs in inflammation and autoimmune regulation, including miRNA, lncRNA, and circular RNA. LncRNA is an emerging area of focus in RA pathology. Many lncRNAs such as MEG3 and LINC00305 are aberrantly expressed in RA. The exploration of their underlying molecular mechanisms will offer a new direction to understand the pathogenesis of RA. It is also promising as a novel diagnostic and therapeutic strategy for RA. Decreased serum levels of lncRNA MEG3 could be attributed to RAassociated hypoxia. LncRNA MEG3 downregulation was associated with increased inflammation, cell proliferation and decreased apoptosis. On the other hand, RA was associated with increased LINC00305 which served as a regulator of inflammatory, hypoxic, invasive, apoptotic and proliferative mechanisms associated with RA.

\section{Conflict of interest}

The authors report no declaration of conflict of interest.

\section{References:}

Al-Rugeebah, A., Alanazi, M., and Parine, N.R., 2019. MEG3: an Oncogenic Long Non-coding RNA in Different Cancers. Pathol Oncol Res,25 (3), 859-874.

Alarcon, G.S., Koopman, W.J., Acton, R.T., and Barger, B.O., 1982. Seronegative rheumatoid arthritis. A distinct immunogenetic disease? Arthritis Rheum,25 (5), 502-507.

Arend, W.P. and Firestein, G.S., 2012. Prerheumatoid arthritis: predisposition and transition to clinical synovitis. Nat Rev Rheumatol,8 (10), 573-586.

Barlow, D.P., 2011. Genomic imprinting: a mammalian epigenetic discovery model. Annu Rev Genet,45 379-403. 


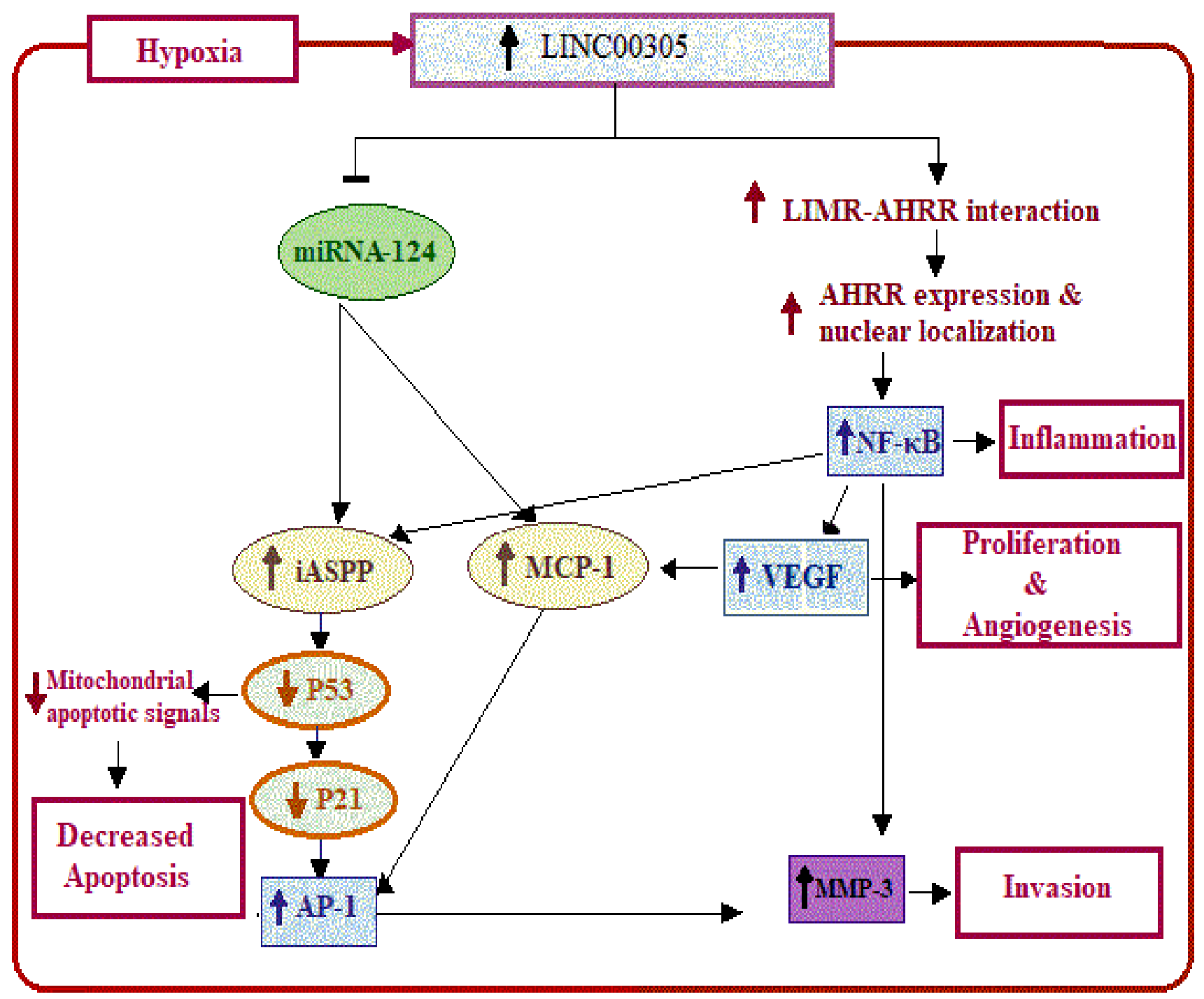

Figure 4: Implication of IncRNA LINC00305 in RA pathogenesis. LIMR= lipocalin-1 interacting membrane receptor, $\mathrm{AHRR}=$ aryl-hydrocarbon receptor repressor, $\mathrm{iASPP}=$ inhibitory member of the apoptosis stimulating protein $\mathrm{p} 53, \mathrm{MCP}-$ $1=$ monocyte chemoattractant protein $-1, \mathrm{NF}-\mathrm{\kappa B}=$ nuclear factor-kappa beta, VEGF = vascular endothelial growth factor, AP-1= activator protein-1, MMP-3 = matrix metalloproteinase-3.

Benetatos, L., Vartholomatos, G., and Hatzimichael, E., 2011. MEG3 imprinted gene contribution in tumorigenesis. Int J Cancer, 129 (4), 773-779.

Biswas, S., Sarabusky, M., and Chakrabarti, S., 2019. Diabetic Retinopathy, lncRNAs, and Inflammation: A Dynamic, Interconnected Network. J Clin Med,8 (7), 1033.
Carrieri, C., Cimatti, L., Biagioli, M., Beugnet, A., Zucchelli, S., Fedele, S., Pesce, E., Ferrer, I., Collavin, L., and Santoro, C., 2012. Long noncoding antisense RNA controls Uchl1 translation through an embedded SINEB2 repeat. Nature,491 (7424), 454-457.

Ceribelli, A., Yao, B., Dominguez-Gutierrez, P.R., Nahid, M.A., Satoh, M., and Chan, E.K., 2011. MicroRNAs in systemic rheumatic diseases. Arthritis Res Ther, 13 (4), 229. 
Chen, S.Y., Shiau, A.L., Wu, C.L., and Wang, C.R., 2017. P53-derived hybrid peptides induce apoptosis of synovial fibroblasts in the rheumatoid joint. Oncotarget,8 (70), 115413-115419.

Chimenti, M., Triggianese, P., Conigliaro, P., Candi, E., Melino, G., and Perricone, R., 2015. The interplay between inflammation and metabolism in rheumatoid arthritis. Cell Death Dis,6 (9), e1887.

Cieślik, M., Czapski, G.A., Wójtowicz, S., Wieczorek, I., Wencel, P.L., Strosznajder, R.P., Jaber, V., Lukiw, W.J., and Strosznajder, J.B., 2020. Alterations of Transcription of Genes Coding Anti-oxidative and Mitochondria-Related Proteins in Amyloid $\beta$ Toxicity: Relevance to Alzheimer's Disease. Mol Neurobiol,57 (3), 1374-1388.

da Rocha, S.T., Edwards, C.A., Ito, M., Ogata, T., and Ferguson-Smith, A.C., 2008. Genomic imprinting at the mammalian Dlk1-Dio3 domain. Trends Genet, 24 (6), 306-316.

Dong, P., Ihira, K., Hamada, J., Watari, H., Yamada, T., Hosaka, M., Hanley, S.J., Kudo, M., and Sakuragi, N., 2015. Reactivating p53 functions by suppressing its novel inhibitor iASPP: a potential therapeutic opportunity in p53 wild-type tumors. Oncotarget,6 (24), 19968-19975.

Guo, Q., Wang, Y., Xu, D., Nossent, J., Pavlos, N.J., andXu, J., 2018. Rheumatoid arthritis: pathological mechanisms and modern pharmacologic therapies. Bone Res, 6 (1), 15.

Hadjicharalambous, M.R. and Lindsay, M.A., 2019. Long non-coding RNAs and the innate immune response. Non-coding RNA,5 (2), 34.

He, C., Yang, W., Yang, J., Ding, J., Li, S., Wu, H., Zhou, F., Jiang, Y., Teng, L., and Yang, J., 2017. Long Noncoding RNA MEG3 Negatively Regulates Proliferation and Angiogenesis in Vascular Endothelial Cells. DNA Cell Biol,36 (6), 475-481.

Hu, F., Mu, R., Zhu, J., Shi, L., Li, Y., Liu, X., Shao, W., Li, G., Li, M., and Su, Y., 2014. Hypoxia and hypoxia-inducible factor- $1 \alpha$ provoke toll-like receptor signalling-induced inflammation in rheumatoid arthritis. Ann Rheum Dis,73 (5), 928936.
Hu, X.Q., Chen, M., Dasgupta, C., Xiao, D., Huang, X., Yang, S., and Zhang, L., 2017. Chronic hypoxia upregulates DNA methyltransferase and represses large conductance $\mathrm{Ca} 2+$-activated $\mathrm{K}+$ channel function in ovine uterine arteries. Biol Reprod,96 (2), 424-434.

Lao, M.-X. and Xu, H.-S., 2020. Involvement of long non-coding RNAs in the pathogenesis of rheumatoid arthritis. Chin Med J,133 (8), 941-950.

Li, H. and Wan, A., 2013. Apoptosis of rheumatoid arthritis fibroblast-like synoviocytes: possible roles of nitric oxide and the thioredoxin 1. Mediat Inflamm,2013, 953462.

Li, J., Song, Q., Shao, L., Zhang, L., Guo, X., and Mao, Y., 2018. MiR-124a inhibits proliferation and invasion of rheumatoid arthritis synovial fibroblasts. Eur Rev Med Pharmacol Sci,22 (14), 4581-4588.

Li, X., Yu, M., Han, L., Chen, L., Zhang, D., Zhou, G., Zhao, Q., and Sun, T., 2019. LINC00305 represses miR-124 expression to trigger inflammatory insults in the presence of lipopolysaccharide. Artif Cells Nanomed Biotechnol,47 (1), 2352-2360.

Liang, J., Chen, W., and Lin, J., 2019. LncRNA: An All-rounder in Rheumatoid Arthritis. J Translat Intern Med,7 (1), 3-9.

Liu, B., Sun, L., Liu, Q., Gong, C., Yao, Y., Lv, X., Lin, L., Yao, H., Su, F., and Li, D., 2015. A cytoplasmic NF- $\mathrm{KB}$ interacting long noncoding RNA blocks I $\mathrm{KB}$ phosphorylation and suppresses breast cancer metastasis. Cancer Cell,27 (3), 370381.

Liu, T., Zhang, L., Joo, D., and Sun, S.-C., 2017. NF- $\kappa B$ signaling in inflammation. Signal Transduct Target Ther,2, 17023.

Liu, Y.R., Yang, L., Xu, Q.Q., Lu, X.Y., Ma, T.T., Huang, C., and Li, J., 2019. Long noncoding RNA MEG3 regulates rheumatoid arthritis by targeting NLRC5. J Cell Physiol,234 (8), 14270-14284.

Lu, X. and Qian, J., 2019. Downregulated MEG3 participates in rheumatoid arthritis via promoting proliferation of fibroblast-like synoviocytes. Exp Ther Med,17 (3), 1637-1642. 
Luo, G., Wang, M., Wu, X., Tao, D., Xiao, X., Wang, L., Min, F., Zeng, F., and Jiang, G., 2015. Long Non-Coding RNA MEG3 Inhibits Cell Proliferation and Induces Apoptosis in Prostate Cancer. Cell Physiol Biochem: International Journal of Experimental Cellular Physiology, Biochemistry, and Pharmacology,37 (6), 2209-2220.

Ma, Y., Zhu, B., Liu, X., Liu, Z., Jiang, L., Wei, F., Yu, M., Wu, F., Zhou, H., and Xu, N., 2017. iASPP overexpression is associated with clinical outcome in spinal chordoma and influences cellular proliferation, invasion, and sensitivity to cisplatin in vitro. Oncotarget, 8 (40), 68365-68380.

Mann, J., 2017. Epigenetics in Liver Disease: Involvement of Oxidative Stress. In: Muriel P (ed) Liver Pathophysiology: Therapies and Antioxidants. Elsevier. pp 199-211.

McInnes, I.B. and Schett, G., 2011. The pathogenesis of rheumatoid arthritis. $\mathrm{N}$ Engl J Med,365 (23), 2205-2219.

Motterle, A., Sanchez-Parra, C., and Regazzi, R., 2016. Role of long non-coding RNAs in the determination of beta-cell identity. Diabetes Obes Metab,18 (Suppl 1), 41-50.

Muller, S. and Radic, M., 2015. Citrullinated Autoantigens: From Diagnostic Markers to Pathogenetic Mechanisms. Clin Rev Allergy Immunol,49 (2), 232-239.

Nakamachi, Y., Kawano, S., Takenokuchi, M., Nishimura, K., Sakai, Y., Chin, T., Saura, R., Kurosaka, M., and Kumagai, S., 2009. MicroRNA$124 \mathrm{a}$ is a key regulator of proliferation and monocyte chemoattractant protein 1 secretion in fibroblast-like synoviocytes from patients with rheumatoid arthritis. Arthritis Rheum,60 (5), 1294 1304.

Ni, S., Li, C., Xu, N., Liu, X., Wang, W., Chen, W., Wang, Y., and van Wijnen, A.J., 2019. Follistatinlike protein 1 induction of matrix metalloproteinase 1,3 and 13 gene expression in rheumatoid arthritis synoviocytes requires MAPK, JAK/STAT3 and NFКB pathways. J Cell Physiol,234 (1), 454-463.

Okamoto, T., Sanda, T., and Asamitsu, K., 2007. $\mathrm{NF}-\kappa \mathrm{B}$ signaling and carcinogenesis. Curr Pharm Des, 13 (5), 447-462.
Qin, G., Kishore, R., Dolan, C.M., Silver, M., Wecker, A., Luedemann, C.N., Thorne, T., Hanley, A., Curry, C., Heyd, L., Dinesh, D., Kearney, M., Martelli, F., Murayama, T., Goukassian, D.A., Zhu, Y., and Losordo, D.W., 2006. Cell cycle regulator E2F1 modulates angiogenesis via p53-dependent transcriptional control of VEGF. Proc Natl Acad Sci U S A,103 (29), 11015-11020.

Qiu, S., Feng, Y., LeSage, G., Zhang, Y., Stuart, C., He, L., Li, Y., Caudle, Y., Peng, Y., and Yin, D., 2015. Chronic morphine-induced microRNA124 promotes microglial immunosuppression by modulating P65 and TRAF6. J Immunol,194 (3), 1021-1030.

Rutenberg-Schoenberg, M., Sexton, A.N., and Simon, M.D., 2016. The properties of long noncoding RNAs that regulate chromatin. Annu Rev Genomics Hum Genet, 17 69-94.

Smolen, J., Aletha, D., and McInnes, I., 2016. Rheumatoid arthritis. The Lancet,388 (10055), 2023-2038.

Strietholt, S., Maurer, B., Peters, M.A., Pap, T., and Gay, S., 2008. Epigenetic modifications in rheumatoid arthritis. Arthritis Res Ther,10 (5), 219.

Su, W., Xie, W., Shang, Q., and Su, B., 2015. The long noncoding RNA MEG3 is downregulated and inversely associated with VEGF levels in osteoarthritis. Biomed Res Int,2015 356893.

Takayama, K., Ishida, K., Matsushita, T., Fujita, N., Hayashi, S., Sasaki, K., Tei, K., Kubo, S., Matsumoto, T., and Fujioka, H., 2009. SIRT1 regulation of apoptosis of human chondrocytes. Arthritis Rheum,60 (9), 2731-2740.

Wahba, A., Ibrahim, M., Mesbah, N., Saleh, S., Abo-Elmatty, D., and Mehanna, E., 2020. Serum LINC00305 expression and its genetic variant rs2850711 are associated with clinical and laboratory features of rheumatoid arthritis. Brit $\mathbf{J}$ Biomed Sci. doi: 10.1080/09674845.2020

Xu, G., Meng, L., Yuan, D., Li, K., Zhang, Y., Dang, C., and Zhu, K., 2018. MEG3/miR-21 axis affects cell mobility by suppressing epithelialmesenchymal transition in gastric cancer. Oncol Rep,40 (1), 39-48. 
Yeung, F., Hoberg, J.E., Ramsey, C.S., Keller, M.D., Jones, D.R., Frye, R.A., and Mayo, M.W., 2004. Modulation of NF- $\kappa B-d e p e n d e n t$ transcription and cell survival by the SIRT1 deacetylase. EMBO J,23 (12), 2369-2380.

Zeisel, M.B., Druet, V.A., Wachsmann, D., and Sibilia, J., 2004. MMP-3 expression and release by rheumatoid arthritis fibroblast-like synoviocytes induced with a bacterial ligand of integrin $\alpha 5 \beta 1$. Arthritis Res Ther,7 (1), R118-R126.

Zhang, B.-Y., Jin, Z., and Zhao, Z., 2017a. Long intergenic noncoding RNA 00305 sponges miR-136 to regulate the hypoxia induced apoptosis of vascular endothelial cells. Biomed Pharmacother,94 238-243.
Zhang, D.-D., Wang, W.-T., Xiong, J., Xie, X.-M., Cui, S.-S., Zhao, Z.-G., Li, M.J., Zhang, Z.-Q., Hao, D.-L., and Zhao, X., 2017b. Long noncoding RNA LINC00305 promotes inflammation by activating the AHRR-NF- $\mathrm{BB}$ pathway in human monocytes. Sci Rep, 7, 46204.

Zhang, X., Wu, N., Wang, J., and Li, Z., 2019. LncRNA MEG3 inhibits cell proliferation and induces apoptosis in laryngeal cancer via miR23a/APAF-1 axis. J Cell Mol Med 23:6708-6719. 